\title{
SU ALCUNE CINQUECENTINE SCIENTIFICHE DELLA BIBLIOTECA DELL'ISTITUTO LOMBARDO
}

\author{
Nota dei mm.ee. FIORENZA DE BERNARDI (*) e \\ GIANNANTONIO SACCHI LANDRIANI $(* *)$
}

(Adunanza del 22 maggio 2014)

\begin{abstract}
SunTO. - Delle 96 cinquecentine di carattere scientifico, presenti nella Biblioteca dell'Istituto Lombardo e recanti segnatura Luini vengono presentate alcune opere di "scienze naturali", scelte sia per le particolarità iconografiche che per l'originalità del testo. Il trattato di anatomia di Andrea Vesalio è presente nella prima edizione del 1542. Le opere di medicina di Jean François Fernel, di Antonio Fracanzani, di Girolamo Fracastoro, il trattato di botanica di Pier Andrea Mattioli e il trattato geografico di Giovan Battista Ramusio sono presenti in edizioni successive, ma sono comunque tutte opere di grande interesse per valutare le conoscenze del tempo come episodi evolutivi della scienza. Alcune sono piuttosto rare e meritano adeguata valorizzazione. I testi meriterebbero un approfondimento critico per rendere accessibili i contenuti oscurati da un tardo latino o da un italiano spesso latinizzante.
\end{abstract}

$* * *$

ABSTRACT. - We introduce some of the 96 books of scientific content, published in the 16th century and belonging to the Istituto Lombardo library. We chose some books in the field of the "natural sciences" both for iconographic peculiarities and for the original content. The library owns the first edition of the anatomy treatise of Andrea Vesalio. The medical books of Jean Francois Fernel, Antonio Fracanzani, Girolamo Fracastoro, the botanical treatise of Pier Andrea Mattioli and the geographical treatise of Giovan Battista Ramusio although in further edition are of great interest in order to evaluate the state of the art and the evolutionary steps of the science. Some books are

(*) Dipartimento di Bioscienze, Università degli Studi di Milano, Italy.

E-mail: fiorenza.debernardi@unimi.it

(*in) Professore Emerito del Politecnico di Milano, Italy.

E-mail: giannantonio.sacchi@polimi.it 
rather rare and precious and deserve to be accurately exploited. Also the texts should be worthy of a critical investigation, overcoming the linguistic difficulties, both for the latin and for italian texts.

\section{INTRODUZIONE}

Le cosiddette "Cinquecentine", opere a stampa del XVI secolo e dei primi anni del XVII secolo, conservate nella Biblioteca dell'Istituto Lombardo sono in gran parte raccolte nella sala LUINI.

In segnatura Luini sono catalogati 863 titoli, dei quali 96 cinquecentine di carattere scientifico.

Una parte di queste sono traduzioni in latino di testi greci. ${ }^{1}$

La classificazione differisce dall'attuale, anche perché molte opere hanno carattere multidisciplinare. Possiamo tuttavia con buona approssimazione collocare tali opere nei seguenti settori: 60 opere riguardano la Medicina e la "farmacologia" (intesa come indicazione di rimedi per la cura di patologie e descrizioni di anatomia umana), 14 opere riguardano la descrizione di piante (soprattutto medicinali), di animali e resoconti di esplorazioni di terre sconosciute, 6 opere riportano argomenti di Astronomia e cosmografia, 9 opere trattano di Matematica e geometria e 7 opere trattano argomenti che, con interpretazione estensiva, potremmo chiamare di Ingegneria e architettura.

Il carattere generale delle opere è «prerinascimentale». La rappresentazione di esseri viventi e non viventi si sofferma con molta attenzione sugli aspetti descrittivi, con solo qualche timido tentativo di elaborazione teorica. Le opere a carattere medico trattano per lo più della cura delle patologie allora più diffuse e temute (sifilide, peste e podagra), mentre sulle cause di queste malattie e sulle modalità di diffusione delle stesse non viene manifestato interesse.

Quasi tutti i libri hanno frontespizi arricchiti dalla cosiddetta "marca" del tipografo e illustrazioni preziose, disegnate ed incise con

1 L'attenzione ai testi antichi di argomento medico nel secolo XVI era infatti molto forte. Le traduzioni contenevano spesso aggiunte ed emendamenti realizzati dall"interprete, spesso nominato nel titolo. Alcuni di questi interpreti erano veri specialisti, come Teodoro Gaza o Cesare Odonus, ai quali va il merito di aver divulgato i testi greci. Tutti questi libri sono privi di illustrazioni. Vedi in bibliografia "Edizioni latine da testi greci”. 
grande accuratezza ed eleganza. Nei volumi divisi in "libri" l'ultima pagina presenta spesso le righe scalate e la dicitura "finis".

\section{GABRIELE FALLOPPIO ED ALTRI TESTI DI MEDICINA}

\subsection{De morbo gallico liber absolutissimum}

De morbo gallico liber absolutissimum (1565) è un interessante testo, costituito da quattro libri legati insieme, stampati in diversi luoghi, tre contemporanei e uno precedente (IT\ICCU\BVEE\002421). Vuole evidentemente riunire tutte le conoscenze e soprattutto le cure della sifilide (così denominata da Girolamo Fracastoro nel 1530) che nei primi anni del '500 ebbe una spaventosa diffusione (Fig. 1).

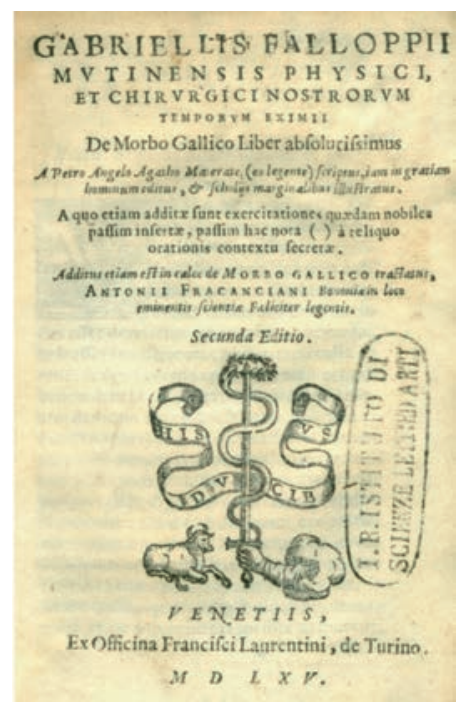

Fig. 1 - Gabriele Falloppio De morbo gallico liber absolutissimum. Frontespizio.

2.2 De morbo gallico fragmenta quaedam elegantissima ex lectionibus anni 1563

Il secondo dei quattro libri legati insieme è: De morbo gallico fragmenta quaedam elegantissima ex lectionibus anni 1563 di Antonio 
Fracanciani o Fracanzani (1506-1567), pubblicato a Padova nel 1563 in appendice all'omonima opera di Gabriele Falloppio (Fig. 2) e più volte ristampato (Bologna 1563, 1564, 1574; Padova 1564; Venezia 1566), come risulta anche dal catalogo SBN (IT \ICCU \BVEE 002421). Redatta in uno stile stringato, l'opera affronta tutti gli aspetti della diagnosi e cura della sifilide e propone l'uso di medicamenti a base di mercurio, nonostante la conoscenza dell'alta tossicità. Fracanzani frequentò l'Università di Padova, dove si laureò in arti e medicina nel 1529; nello stesso anno iniziò la carriera accademica nell'ateneo patavino come professore di logica per poi passare, nel 1538, all'insegnamento della medicina teorica come lettore straordinario. Ricordato dai contemporanei come uno dei più brillanti medici dell'epoca, il Fracanzani fu spesso chiamato al capezzale di principi e di illustri personaggi; di lui si lodava la rapidità e l'esattezza nelle diagnosi, nonché l'efficacia delle cure. La fama raggiunta fu certamente all'origine dei riconoscimenti accademici grazie ai quali nel $1546 \mathrm{fu}$ nominato professore ordinario di medicina pratica.

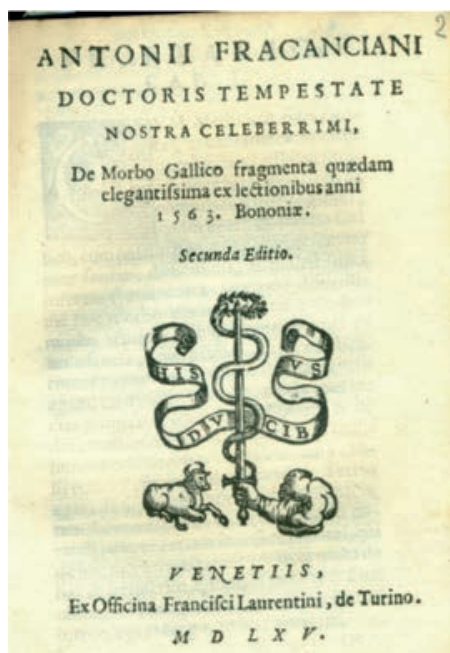

Fig. 2 - Antonio Fracanzani: frontespizio dell'appendice al libro di Falloppio.

\subsection{Mali galeci sanandi}

Il terzo libro, di Tommaso Giannotti Rangoni, detto Thomae Ravenna, ha il promettente titolo "Mali galeci sanandi" (IT\ICCU $\backslash$ 
UBOE\073342) (Fig. 3). Nacque a Ravenna nel 1493 da una famiglia borghese e probabilmente agiata, si laureò a Bologna in medicina e arti e portò per tutta la vita il nomen "Philologus" probabilmente per sottolineare la sua formazione umanistica. Esercitò come medico e insegnante di filosofia e astrologia a Roma e Modena, acquisì grande notorietà per la medicina astrologica e i "pronostici diluviali". Morì a Venezia nel 1577.

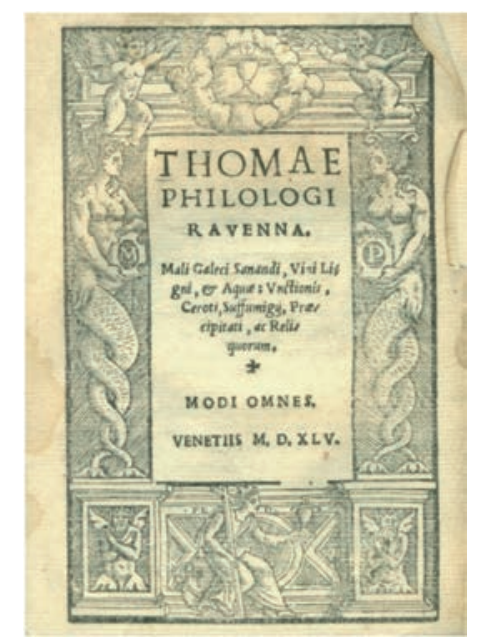

Fig. 3 - Tommaso Giannotti Rangoni: Mali galeci sanandi frontespizio del libro legato insieme ai precedenti.

\subsection{De lue hispanica sive morbo gallico}

Il quarto libro, di Ferrerio Tolosatis (Auger Ferrier 1513-1588), medico di Caterina de' Medici, reca il curioso titolo "De lue hispanica sive morbo gallico", nuova denominazione della malattia (IT \ICCU $\backslash$ MILE \030890) (Fig. 4). Ferrier fu medico, astrologo e matematico. Studiò anche giurisprudenza e divenne esperto in astrologia giudiziaria, disciplina all'epoca molto in voga. Questi due libri non risultano nel catalogo SBN come appendici dei precedenti: probabilmente sono stati legati insieme in epoca successiva alla pubblicazione forse per praticità oppure per riunire fisicamente in un unico testo tutte le conoscenze dell'epoca su questa temutissima malattia. 


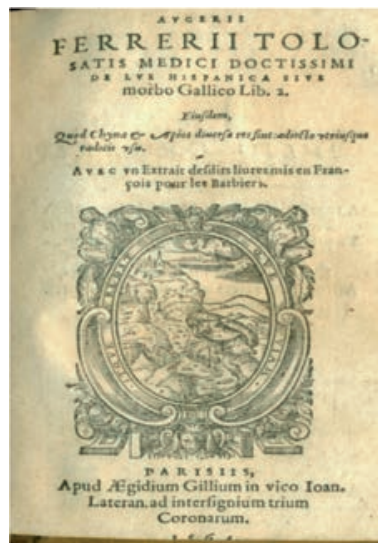

Fig. 4 - Auger Ferrier: De lue hispanica sive morbo gallico, frontespizio del libro legato insieme ai precedenti.

2.5 Opera Genuina Omnia in tres tomos distributas, III edizione, postuma, del 1606

Di Gabriele Falloppio (Modena 1523- Padova 1562) la biblioteca conserva anche l'Opera Genuina Omnia in tres tomos distributas, III edizione, postuma, del 1606 (IT\ICCU\UFIE $\backslash 000445$ ) (Fig. 5).

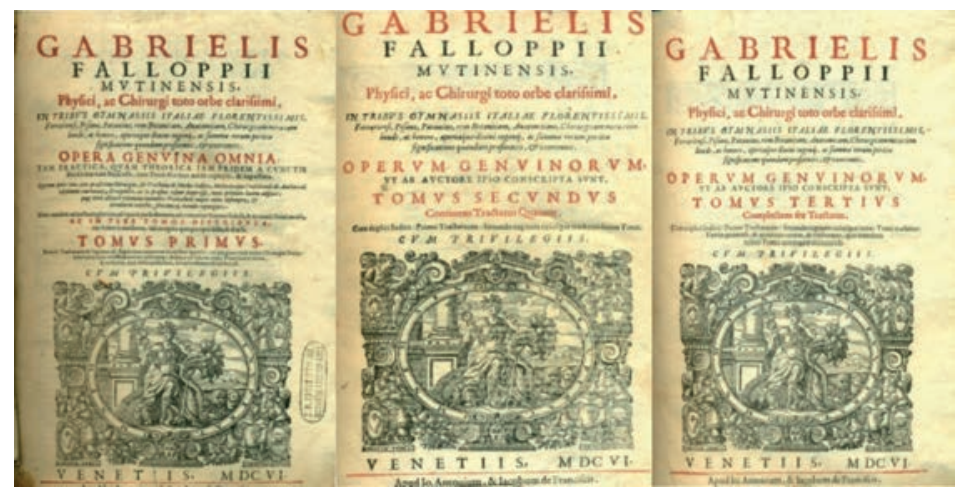

Fig. 5 - Gabriele Falloppio Opera Genuina Omnia in tres tomos distributas, III edizione, postuma, 1606.

E' costituita per lo più da una serie di lezioni tenute a Padova, nelle quali dà esempio della sua competenza come medico, chirurgo e 
splancnologo e delle sue conoscenze di botanica, farmacologia e idrologia. Sono note infatti le sue descrizioni degli organi riproduttivi femminili (le tube uterine note come tube del Falloppio). La descrizione dell'anatomia dei feti e dei neonati, posta in relazione con quella degli adulti, costituì il prodromo dell'embriologia. Le numerose dissezioni e descrizioni anatomiche di animali furono il fondamento dell'anatomia comparata. Il suo allievo Fabrizio d'Acquapendente è considerato infatti uno dei fondatori dell'embriologia (molto nota è la borsa di Fabrizio degli uccelli).

Il primo volume di questa terza edizione postuma (1606) è preceduta da una Medici intimi Epistola (1585) di Giovanni Crato von Krafftheim, medico ed umanista tedesco di grande fama, molto legato a Martin Lutero (Fig. 6). In essa si danno consigli su medicamenti, acque termali, metalli e fossili, per curare malattie endemiche come la peste (della quale fu tra i primi a riconoscere la contagiosità) il catarro, la podagra. Sembra che l'autore abbia richiesto l'illustre presentazione (caso non infrequente) per accreditare ulteriormente la sua opera, già molto importante.

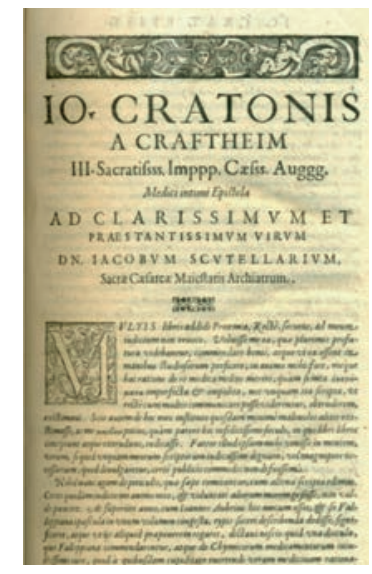

Fig. 6-Giovanni Crato von Krafftheim Medici intimi Epistola 1585.

\subsection{Universa medicina}

Il trattato "Universa medicina" di Jean François Fernel (14851558) è presente nel fondo Belloni e contrassegnato da un prezioso "ex libris" (IT \ICCU \MILE $\backslash 014705$ ); la prima edizione fu stampata a Parigi nel 1554, questa dovrebbe essere una edizione successiva e 
porta la data, scritta a mano, del 1619 (Fig. 7). Sebbene la data, la marca e la città coincidano, il nome del tipografo, apud Petrum E Iacobum Chouet, non è quello riportato nel catalogo SBN: Stephanus Gamoetus. Si sa che il trattato di patologia e terapia fu ristampato più volte. Fernel studiò a Parigi medicina, matematica e astronomia,fu uno dei più dotti rappresentanti della medicina galenica modificata dalle dottrine arabe. Diede contributi molto importanti alla medicina e introdusse nel lessico medico il termine "fisiologia" per descrivere lo studio funzionale del corpo. La sua straordinaria erudizione generale, l'abilità e il successo con cui ha cercato di far rivivere lo studio delle opere dei medici dell'antica Grecia, gli procurarono una grande reputazione, e infine l'ufficio di medico di corte di Enrico II di Francia.

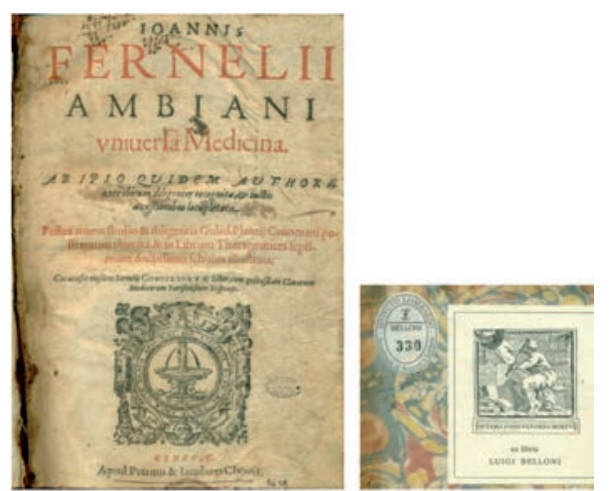

Fig. 7 - Jean François Fernel Universa Medicina con data scritta a mano 1619 e relativo ex libris e numero di inventario dal Fondo Belloni.

\subsection{Opera quae extant, veterum codicum collectione restituta}

Il testo di Girolamo Mercuriale (Forlì 1530-1606) laureato a Padova, cultore delle opere di Ippocrate, è una riedizione dell'opera di Ippocrate in greco ed in latino: Opera quae extant, veterum codicum collectione restituta, pubblicata da Giunta a Venezia nel 1588 (IT \ICCU $\backslash$ BVEE $\backslash 004554)$ con un bellissimo frontespizio formato da 12 piccoli quadri che raffigurano il medico, in abiti cinquecenteschi, nell'esercizio della sua arte. L'edizione latina presenta aggiunte originali del Mercuriali, il quale fu professore di medicina pratica a Padova e a 
Bologna e per primo teorizzò l'uso della ginnastica e della balneoterapia su base medica.

\section{TESTI DI ANATOMIA}

\subsection{De bumani corporis fabrica libris septem}

Una preziosità della biblioteca è "De humani corporis fabrica libris septem" di Andrea Vesalio (Bruxelles 1514-Zante 1564), prima edizione

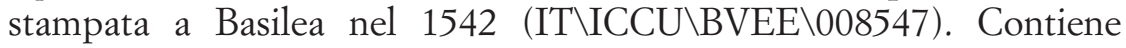
descrizioni molto accurate di muscoli e ossa umane. E' corredato da più di 300 illustrazioni in xilografia opera di van Calcar, un disegnatore e pittore che lavorava nella bottega di Tiziano. Vesalio è considerato il fondatore dell'anatomia moderna, realizzata grazie alle numerose dissezioni di cadaveri a cui si dedicò a Padova e Bologna, dove insegnò (Fig. 8). Nel frontespizio a tutta pagina (Fig. 9) è infatti raffigurata la scena della dissezione del cadavere di una donna nel teatro anatomico, con molti particolari, anche inquietanti, che ricordano la precisione descrittiva dei pittori fiamminghi. Vesalio stesso è raffigurato in una illustrazione (Fig. 10), così come sono raffigurati, ordinati su un tavolo di legno molto simile ad un banco di officina, gli strumenti dello studioso di anatomia: forbici, coltelli, pinze, martelli etc. (Fig. 11).

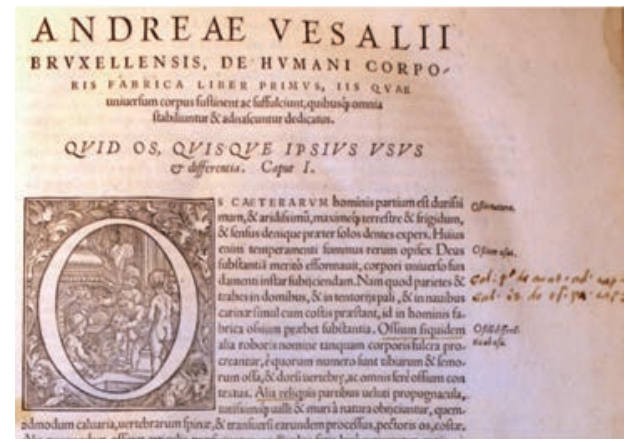

Fig. 8-Andrea Vesalio: De humani corporis fabrica prima pagina del libro primo, con un'annotazione a mano.

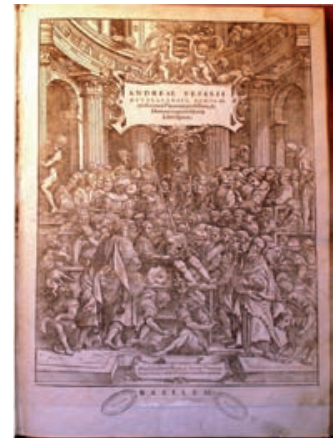

Fig. 9 - Andrea Vesalio: De humani corporis fabrica frontespizio raffigurante la dissezione del cadavere di una donna in un teatro anatomico. 


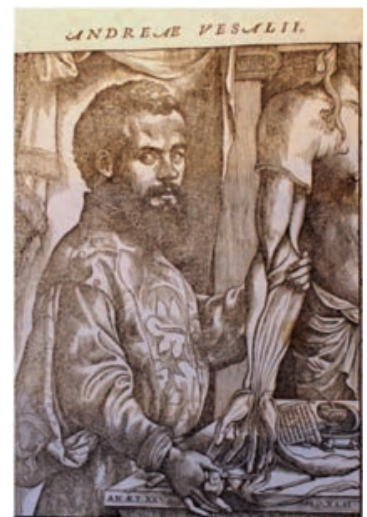

Fig. 10 - Ritratto di Andrea Vesalio.

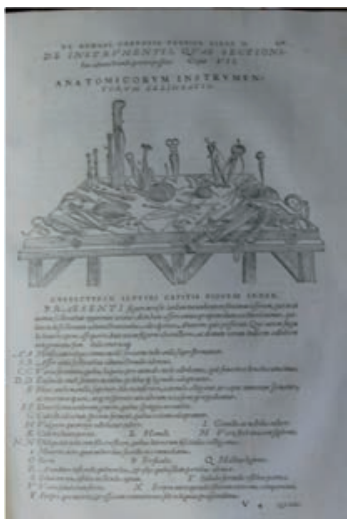

Fig. 11 - Andrea Vesalio: gli strumenti dello studioso di anatomia.

\subsection{Opera omnia di Girolamo Fracastoro}

L'Opera omnia di Girolamo Fracastoro (Verona 1476-1553) (IT\ICCU \TO0E\003410) medico, filosofo, umanista, fu stampata postuma nel 1555, pubblicata dai Giunta di Venezia con successive ristampe del 1574 e del 1584. Il testo conservato nella biblioteca porta l'indicazione "Ex Tertia Editione". Fracastoro è forse più noto come astronomo e geografo, fu amico di Paolo Ramusio (padre di Giovan Battista, il quale gli dedicò la sua opera). Di lui si ricorda anche che fu amico di Copernico e che progettò un cannocchiale, realizzato molto più tardi da Galileo. Fracastoro è un esempio dell'uomo colto dai molteplici interessi, letterari e scientifici: scrisse un poemetto in esametri (Syphilis sive morbus gallicus) nel quale narrava elegantemente del pastore Sifilo affetto da questa terribile malattia, che poi prese da lui il nome.

\section{Piante medicinali E NON SOlO}

\subsection{Historia Plantarum et Vires ex Dioscoride, Paulo Aegineta,} Theophrasto, Plinio et recentioribus greci iuxta elementorum ordinem

Interessante opera con reminiscenze antiche è la "Historia Plantarum et Vires ex Dioscoride, Paulo Aegineta, Theophrasto, Plinio et recentioribus greci iuxta elementorum ordinem" di Konrad von Gessner, 
(Zurigo 1516-1565) professore di greco e archiatra. E' un catalogo di piante in ordine alfabetico molto interessante per chi voglia osservare, conoscere e classificare. Merito del Gessner è di avere attirato l'attenzione sul fiore e sul frutto come caratteri diagnostici. Si ha notizia di molte figure, non presenti nella nostra biblioteca. Fu un grande erudito, tentò di fondare una biblioteca con tutti i libri esistenti (da collezioni della biblioteca vaticana, da cataloghi di stampatori e venditori di libri, una specie di database del XVI secolo. Curiosamente l'opera di Gessner è legata insieme ad un'edizione del volume di Teofrasto (IV sec a.c.), tradotto in latino da Teodoro Gaza, (Tessalonica 1415-1475) umanista ed insegnante di greco a Palermo, Pisa, Pavia, Ferrara. Gaza si specializzò nella traduzione di testi scientifici, spesso emendandoli, ma facendo conoscere le opere zoologiche di Aristotele e le opere botaniche di Teofrasto. Il legare insieme le due opere può avere un duplice significato: una specie di avallo da parte del riconosciuto maestro greco e un confronto con la classificazione di Teofrasto in erbe, frutti, suffrutici. ${ }^{2}$

\subsection{Commentarii in VI libros Pedacii Dioscoridis Anarzabei de Medica materia}

A Dioscoride si rifà anche l'opera di Pietro Andrea Mattioli (Siena 1501-Trento 1578) Commentarii in VI libros Pedacii Dioscoridis Anarzabei de Medica materia in 2 volumi (IT \ICCU $\backslash B V E E \backslash 014932$ ). In realtà si tratta di una traduzione con molte aggiunte dell'opera di Dioscoride, medico greco attivo a Roma nel I secolo dopo Cristo (Fig. 12).

Fu scritto in italiano (1544): Mattioli scelse la lingua italiana, (anzi toscana, come l'autore stesso dichiara) per mettere alla portata di medici e speziali, che per lo più ignoravano le lingue classiche, le conoscenze degli antichi. Una decina di anni più tardi fu tradotto in francese, boemo, tedesco, latino. Grande novità, che spiega la grande diffusione dell'opera, fu l'aggiunta di figure disegnate da Giorgio Liberale, sotto la vigile guida del Mattioli stesso.

Il volume, datato 1583, è una delle 6 ristampe postume, divise in due volumi, che l'editore Valgrisi fece autorizzato dagli eredi Mattioli,

2 Il libro è privo di frontespizio. Anno e luogo di pubblicazione non sono chiaramente definibili. 
riutilizzando le xilografie eseguite da una équipe di artigiani boemi. Si tratta di una summa delle nozioni di medicina naturale del tempo, integrata dalle nozioni da lui stesso apprese nell'attività terapeutica e da notizie sulle virtù di piante importate dalle Indie Orientali e Occidentali e di altre da lui raccolte in Val di Non e sul Monte Baldo. Infatti, opportunamente il Mattioli scrive nel frontespizio stesso " $A b$ ipso autore recogniti et locis plus mille aucti”. Anche se l'intento del Mattioli medico era sicuramente quello di descrivere le proprietà medicinali delle piante, il testo è diventato il più importante libro di Botanica fino al Systema naturae di Linneo (1735).

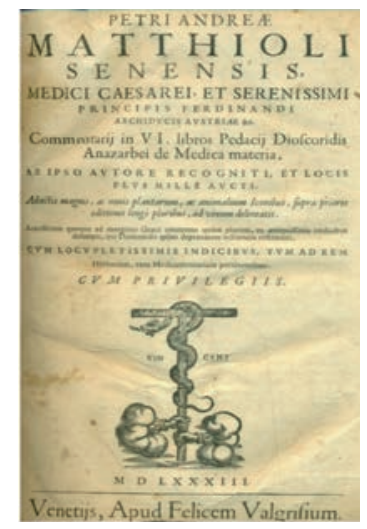

Fig. 12 - Pietro Andrea Mattioli: Commentarii... frontespizio del primo volume.

Nelle figure viene spesso privilegiata la rappresentazione artistica, a discapito della precisione scientifica; ad esempio, il portamento della chioma viene spesso esaltato con simmetrie improbabili; la forma e gli intrecci delle radici vengono accentuati secondo l'ispirazione dell'artista più che secondo il rigore descrittivo (Figg. 13, 14). Il Mattioli fu oggetto di polemica da parte dell'illustre botanico prussiano Melchior Wieland detto il Guilandino, che lo accusò di errori nell'identificazione di specie botaniche (1557). L'illustre botanico Konrad Gessner, però, lo difese e, tre anni dopo, Giovanni Crato von Krafftheim, il già ricordato autorevole medico ed umanista tedesco, pose fine alla diatriba sostenendo in via definitiva che gli errori non sminuivano il valore dell'opera ed inviò una lettera consolatoria al Mattioli. La polemica ebbe ancora qualche strascico fino al 1562 e può essere assunta come esempio della vivacità polemica e della velocità di diffusione dei libri e delle idee, non- 
ché della rete di connessione tra gli eruditi, favorevole ad attiva comunicazione.

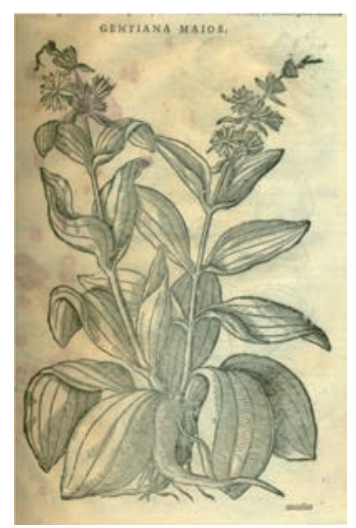

Fig. 13 - Pietro Andrea Mattioli:

Commentarii... pianta di genziana.

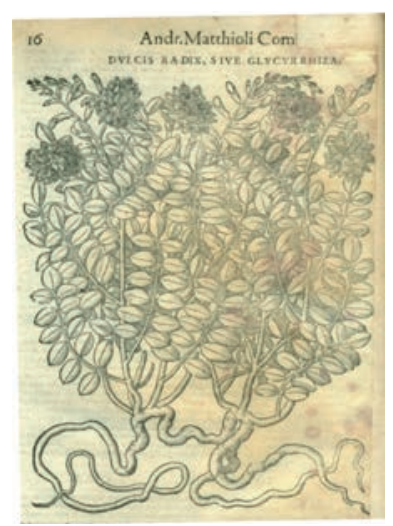

Fig. 14 - Pietro Andrea Mattioli:

Commentarii... pianta di liquirizia.

Nel secondo volume sono riportate immagini e descrizioni di animali selvatici e domestici, e tecniche di allevamento di questi ultimi. Sono inoltre descritte proprietà curative, piuttosto curiose, di sostanze tratte da animali, ne presentiamo due esempi (Figg. 15,16):

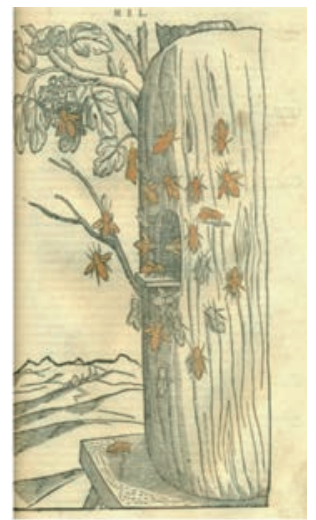

Fig. 15 - Pietro Andrea Mattioli: Commentarii... volume secondo: favo di api.

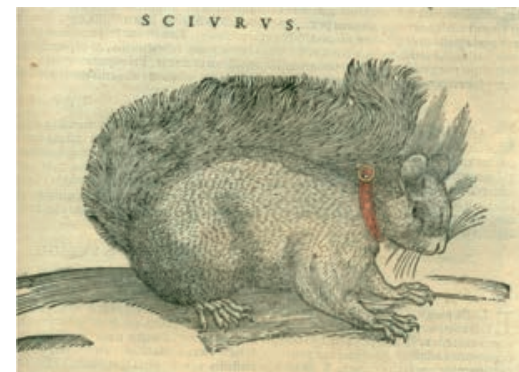

Fig. 16 - Pietro Andrea Mattioli: Commentarii... volume secondo: scoiattolo. 
Il miele è utile, mescolato al salgemma, per curare tonsille, tosse, veleni di funghi e di cani rabbiosi, ferite varie e anomalie cutanee.

Il grasso di scoiattolo, mescolato con aceto, riduce la caduta dei capelli. La carne è cibo utile e per "palati non volgari".

\section{LE GRANDI SCOPERTE GEOGRAFICHE}

Di grandissimo interesse storico è il trattato "Delle navigazioni et viaggi" di Giovan Battista Ramusio: è il primo trattato geografico dell'età moderna, dedicato a Girolamo Fracastoro, amico del padre: fu pubblicato tra il 1550 e il 1606 in varie edizioni (quella dell'Istituto Lombardo è del 1563; IT \ICCU $\backslash P U V E \backslash 000097$ ); riunisce una cinquantina di memoriali di viaggi ed esplorazioni dall'antichità classica fino al suo tempo, che coprono tutto i mondo allora conosciuto. Particolarmente interessanti sono le descrizioni e le raffigurazioni di piante, animali e manufatti sconosciuti, che gli esploratori trovarono nelle terre visitate. Ramusio era un umanista, geografo e raffinato diplomatico della Repubblica Veneta, venne inviato come ambasciatore presso parecchie corti europee. Tramite le sue amicizie diplomatiche ottenne i resoconti di vari esploratori e, su queste basi, fu molto abile nel disegnare le mappe di territori che non aveva mai visitato, come la mappa, qui raffigurata, dell'Isola Spagnuola, su cui mise piede Cristoforo Colombo dopo aver attraversato l'Atlantico. La Fig. 17 è tratta dalla Storia delle Indie Occidentali di Gonzalo Fernando Doviedo nel volume terzo.

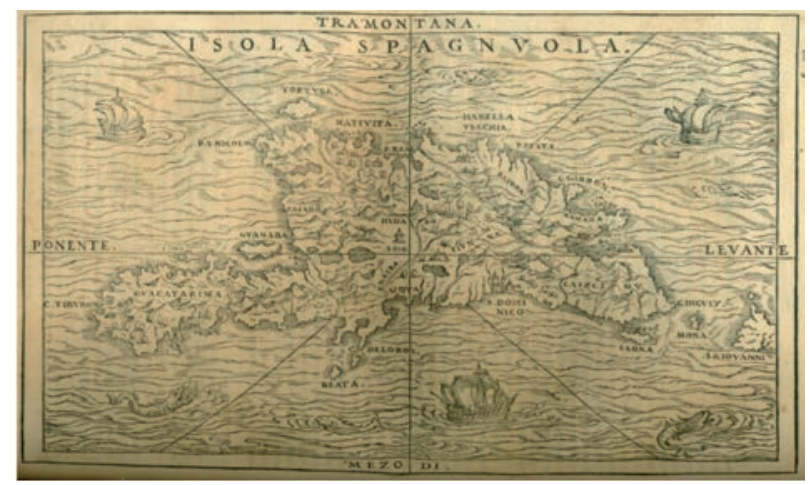

Fig. 17 - Giovan Battista Ramusio: Delle navigazioni et viaggi. Volume terzo: mappa dell'isola Spagnuola. 
Nel primo volume sono riportati viaggi in Africa, Indie Occidentali, Perù, e nei territori scoperti da Cristoforo Colombo, Fernando Cortese, Francesco Pizzarro, IndieOrientali, Calcutta, Giapan, Terra di Prete Ianni.

Nel secondo volume: Istoria delle cose de Tartari e diversi fatti de loro Imperatori descritta da M. Marco Polo gentilhuomo venetiano. Nel terzo volume sono riportate relazioni Su le navigazioni al Mondo Nuovo agli antichi Incognito fatto da don Cristoforo Colombo Genovese...... in diverse parti delle Indie occidentali. Nel terzo volume sono anche belle illustrazioni che raffigurano piante, animali e manufatti che gli esploratori trovarono nell'isola Spagnuola e descrissero.

E' interessante leggere le brevi frasi che aprono alcuni paragrafi e che fanno da didascalia alle figure (Figg. 18-21).

"Del pane degli Indiani chiamato Mabiz, e come questo frumento si semina e si raccoglie... (mais a 8 file) .... Nasce il Mabiz in certe canne, che nascono e pullulano, con mazzocche d'un palmo....e sono piene di granelli grossi come ceci, ma non tondi del tutto". La descrizione è piuttosto accurata ed è seguita da una parimenti accurata descrizione di come gli abitanti seminano i granelli nel terreno dopo averli lasciati in acqua per due giorni.

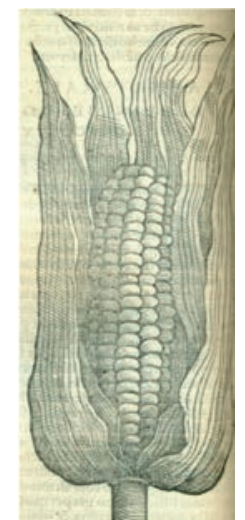

Fig. 18 - Giovan Battista Ramusio:

Delle navigazioni et viaggi. Volume terzo: spiga di Mahiz.

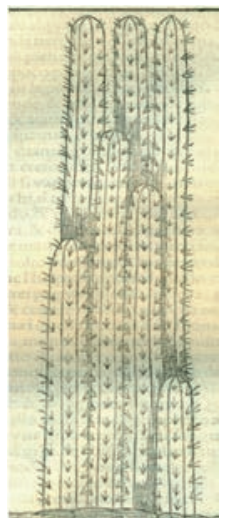

Fig. 19 - Giovan Battista Ramusio:

Delle navigazioni et viaggi. Volume terzo: Cereus, cactacea.

Di certi cardi alti e diritti, come picche lunghi, quadri e spinosi, chiamati Cery dai cristiani, perché paiono ceri o torchi di cera, fuori che nelle 
spine... (Nella sistematica attuale le cactacee comprendono il genere Cereus, mentre la figura si riferisce probabilmente alla specie Pachycereus pringlei, la più alta delle cactacee).

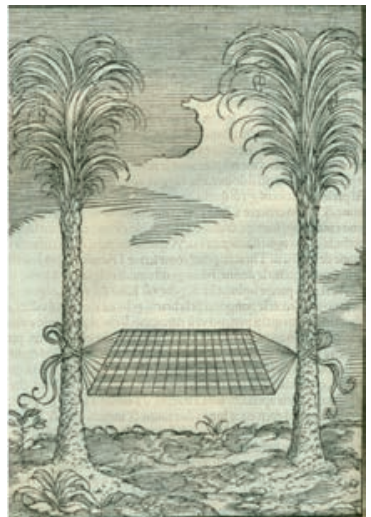

Fig. 20 - Giovan Battista Ramusio:

Delle navigazioni et viaggi.

Volume terzo: amaca.

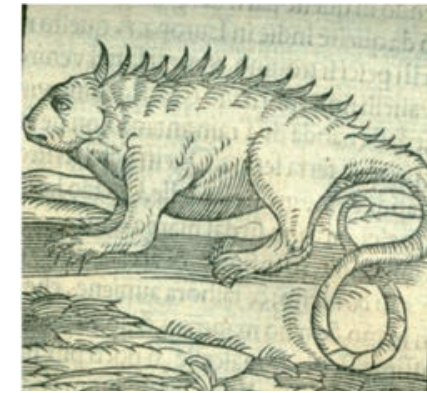

Fig. 21 - Giovan Battista Ramusio:

Delle navigazioni et viaggi. Volume terzo: iguana.

...che maniere di letto hanno in questa isola (Spagnuola). Un lenzuolo in parte tessuto, a modo di una rete, che più fresco sia.

Delle Iuana serpentes il quale si tiene neutrale, cioè in dubbio se carne o pesce perché va per li fiumi e per gli alberi medesimamente.

\section{OSSERVAZIONI E CONGETTURE}

Le opere presentate consentono la valutazione comparativa tra le conoscenze del XVI secolo e quelle dei secoli che seguono. Nel loro insieme contengono una magia. Sono l'inizio del travolgente successo della nascente editoria quale diffusore del sapere e preludono all'intensa evoluzione del pensiero scientifico. Uno sguardo superficiale a quanto prodotto nel secolo successivo consente di sottolineare il passaggio dalla descrizione anatomica alla morfologia funzionale, per esempio dall'osservazione empirica dell'utilità di un medicamento alla sperimentazione basata su ipotesi logiche.

Gli autori del tempo, spesso anche letterati e scrittori, possedevano una vasta cultura umanistica. La conoscenza del greco antico era indispensabile per esaminare, riportare ed emendare gli antichi testi ai quali 
molti facevano riferimento. Non pochi si fregiavano del titolo di "Philosophus" per poi dedicarsi alla medicina, alla descrizione di piante medicinali, ai metodi di estrazione dei principi medicamentosi. Alcuni erano anche astronomi sostanzialmente d'impostazione tolemaica.

L'astrologia era loro compagna di viaggio quale mezzo empirico e seducente per misurare il tempo. Gli orologi si sarebbero affermati e perfezionati soltanto nei secoli successivi.

Tutti i libri presentati mostrano una veste editoriale di grande bellezza: gli editori manifestano un gusto sopraffino nella scelta delle marche, spesso elaborate e cariche di simbologia, nella composizione dei frontespizi, nella composizione delle ultime pagine dei libri. Attenzione particolare meritano le illustrazioni. Splendide e pensate come quadri sono le figure in xilografia di Jan Stephan Calcar per il testo anatomico di Vesalio. Di notevole precisione, non disgiunta da ricerca artistica, sono le xilografie boeme dell'opera botanica di Mattioli. La bellezza della veste grafica contribuì a rendere quell'opera il più grande classico di botanica. Gli studiosi attribuiscono parte del merito della diffusione all'esecuzione e composizione delle figure. Una buona parte della fortuna dell'editore Valgrisi è attribuibile al testo illustrato del Mattioli.

Alcuni libri sono rari: di De lue hispanica di Ferrier Auger è segnalata solo una copia in Italia, nella Biblioteca Braidense; i testi di botanica di Gessner e di Mattioli, il secondo volume delle opere compilative di Ramusio e di Falloppio, risultano uniche nel panorama bibliotecario milanese.

Tutti i testi presentati meriterebbero un'adeguata valorizzazione storica e un approfondimento critico. La lettura presenta notevoli difficoltà, soprattutto per i testi in latino, dove la lingua viene utilizzata per illustrare concetti diversi dall'uso classico: la collaborazione tra cultura umanistica e scientifica può ottenere risultati di grande interesse nel tracciare un cammino evolutivo della scienza.

Quando si affrontano catalogazioni critiche, come nel percorso al quale appartiene questo saggio, ci si può domandare quale obiettivo si persegua. La domanda è meno peregrina di quanto s'immagini a prima vista. La risposta, forse ambigua, è che la finalità concettuale nasce e si forma durante l'elencazione del materiale bibliografico di cui si dispone. Alla curiosità per l'antico splendore subentra una sorta di reverenziale rispetto per la fatica di uomini antichi, corredati da possibilità d'informazione ben più povere delle nostre. Tanto grandi da lanciare messaggi, che portano i segni del loro ingegno e del loro mondo, nel nostro tempo. Noi 
crediamo nella preziosa indicazione morale dell'antica fatica. Deve essere ancora da noi militata, perché il futuro, con la pazienza del tempo, ci assista nell'accedere a valori umani sempre più elevati.

\section{BIBLIOGRAFIA}

Andreoli Ilaria. Ex officina erasmiana. Vincenzo Valgrisi e l'illustrazione del libro tra Venezia e Lione alla metà del ' 500 . Tesi di dottorato in cotutela Università di Ca' Foscari, Venezia e Université Lumière Lyon 2, 2006.

Belloni Speciale Gabriella. Falloppia Gabriele in Dizionario Biografico degli italiani. Vol 44, Treccani 1994.

Fausti Daniela. Su alcune traduzioni cinquecentesche di Dioscoride: da Ermolao Barbaro a Pietro Andrea Mattioli. in Sulla tradizione indiretta di testi medici greci: le traduzioni. Atti del III seminario internazionale. (I. Garofalo, S. Fortuna, A. Lami, A. Roselli eds) Certosa di Pontignano 18-19 settembre 2009.

Hesse Hermann. Le poesie dello studente in Il gioco delle perle di vetro, traduzione di Ervino Pocar. Mondadori, Milano 1955.

Preti Cesare. Mattioli Pietro Andrea. In Dizionario Biografico degli italiani. Vol 72 Treccani 2008.

Ramusio G.B. Navigazioni e viaggi 6 voll, a cura di Marica Milanesi. Einaudi "I millenni"1988.

Sacchi Landriani G.A. Cinquecentine di argomento matematico. Istituto Lombardo Rendiconti Scienze, 147, 2013.

Edizioni latine da testi greci

Aristotele. Aristotelis Stagiritae principi de Historia animalium. Theodoro Gaza interprete. Historia animalium, De partibus animalium, De generatione animalium. De plantis. Venetiis 1585 .

Dioscoride. Dioscoridis et Stephani atheniensis Alphabetum empiricum a Casparo Vuolpio tigurino medicum in lingua latina conversus, 1581.

Teofrasto. Theophrasti sparsae de plantis sententiae in continuatam seriem ad propria capita revocatae, nominaque secundum literarum ordinem disposita per Cesarem Odonum Philosophum ac Medicum Bononiae praticam medicinae ordinariam profitentem. Bononiae apud Alexandrum Benaccium, 1561.

Ippocrate. Hippocratis Epidemiorum liber sextus. Traduzione latina, 1582, legato insieme a Claudi Galeni pergamena liber de optima secta ad thrasibulum. Iunio Paulo patavino interprete.

Ippocrate. Hippocratis Coi Opera per Ianum Cornarium latina lingua conscripta. Venetiis apud Hieronymus Scotum 1546.

Paolo Egineta. Pauli Aeginetae De Arte Medendi libri VII. Bernardo Feliciano interprete. Venetiis in aedibus Luceantonii Iuntae Florentini 1532. 